\title{
Effect of Inspiratory Muscle Training on Performance of Handball Athletes
}

\author{
by \\ Charlini S. Hartz', Márcio A. G. Sindorf', Charles R. Lopes ${ }^{1,2}$, José Batista1, Marlene \\ A. Moreno ${ }^{1}$
}

Inspiratory muscle training (IMT) is a strategy that has been used to improve performance in different sports modalities. This study investigated the effects of an IMT program on respiratory muscle strength and resistance as well as aerobic physical performance (PP) of handball athletes. Nineteen $20 \pm 3$ year-old male athletes were allocated into an experimental ( $E G, n=10)$ or a placebo group $(P G, n=9)$. Their respiratory muscle strength was evaluated by measuring the maximum inspiratory and expiratory pressures (MIP and MEP), muscular respiratory resistance by maximum voluntary ventilation (MVV) and aerobic PP by the cardiopulmonary exercise test. The study was designed to evaluate the effects of a 12-week IMT program with five sessions a week. A significant difference was observed in the pre and post IMT values of the MIP (170 \pm 34 to $262 \pm 33 \mathrm{cmH} 2 \mathrm{O})$ and MEP (177 \pm 36 to $218 \pm 37 \mathrm{cmH} 2 \mathrm{O})$ in the $E G$, and MIP (173 \pm 45 to $213 \pm 21 \mathrm{cmH} 2 \mathrm{O})$ in the $P G$, with a large effect size for the MIP, when the groups were compared. MVV showed a significant increase (162 \pm 24 to $173 \pm 30 \mathrm{~L})$ in the EG, with a small effect size. There was a significant difference in maximum oxygen uptake ( $54 \pm 8$ to $60 \pm 7 \mathrm{ml} / \mathrm{kg} / \mathrm{min}$ ) in aerobic PP. Oxygen uptake at the respiratory compensation point $(R C P)(46 \pm 6$ to $50 \pm 5 \mathrm{ml} / \mathrm{kg} / \mathrm{min})$, with a moderate effect size for both variables, was observed in the EG after IMT. We concluded that IMT provided a significant increase in respiratory muscle strength and resistance, contributing to increased aerobic PP in the EG, which suggests that IMT could be incorporated in handball players' training.

Key words: respiratory muscles, athletic performance, sports, aerobic capacity.

\section{Introduction}

The primary function of the respiratory system is to maintain alveolar ventilation, this being proportional to the metabolic needs (Janssens et al., 2013). In high intensity exercises, maintained for a prolonged period, respiratory muscle performance decreases in response to increased respiratory muscle work and dyspnea, which will contribute to respiratory muscle fatigue and a decrease in global performance (Wells and Norris, 2009). The decreased respiratory function can be associated with and is suggested to compromise performance during heavy exercise, due to the activation of the metaboreflex mechanism, which, in response to inspiratory muscle fatigue and dyspnea, increases the blood flow to the respiratory muscles, while decreasing the blood flow to the activated peripheral musculature (Romer and Polkey, 2008). Inspiratory muscle training (IMT) has been used as a strategy to minimize respiratory fatigue, and this is why it is considered to be a method that has ergogenic potential in athletic performance.

Benefits from IMT are related to

\footnotetext{
1 - Postgraduate Program in Human Movement Sciences - Methodist University of Piracicaba - UNIMEP - Piracicaba, São Paulo, Brazil.

2 - Adventist College of Hortolândia, Hortolândia, São Paulo, Brazil.
} 
increased strength and resistance of the respiratory musculature, once they can enhance sport performance in athletes, especially in modalities with high metabolic demands (HajGhanbari et al., 2013). Handball is considered to be a sport of high intermittent intensity, generated by large mechanical and metabolic demands, since $63 \%$ of the game consists of execution of jumps, sprints and movements of attack and defense at high intensity, for about 32 to 53 minutes during the game, by each player, which shows the need for the anaerobic and aerobic metabolism in this modality (Karcher and Buchheit, 2014). Wagner et al. (2014) showed in a review that $\mathrm{VO} 2 \mathrm{max}$ in elite and experienced team-handball players should be around 55 $\mathrm{ml} \cdot \mathrm{kg}-1 \cdot \mathrm{min}-1$. Thus, training methods used in these athletes have the objective to reduce the effect of fatigue, for greater intensity and duration of performance during the game (Povoas et al., 2014).

Recent investigations have indicated that in high intensity modalities that frequently use the upper limbs, such as rowing and swimming, the overload on the respiratory musculature is even higher. In these modalities, the double demand on the inspiratory muscles occurs because these muscles require maintenance of high ventilatory demands. In addition, they are recruited to perform sport specific activities during the game. These facts culminate in a double demand on the inspiratory muscles, since these muscles are involved in the mechanical actions during inspiration, and also recruited during upper limb movement, increasing the susceptibility to greater fatigue and consequent failure in muscle and physical performance (Lomax et al., 2014).

Considering the effect of IMT on performance of athletes, and the need for methods that reflect greater yields during a game, the objective of this study was to investigate whether 12 weeks of IMT could increase respiratory muscle strength, as well as improve aerobic performance of handball athletes.

\section{Methods}

The study was designed to evaluate the effects of a 12-week IMT program on respiratory muscle strength and resistance, as well as aerobic performance of handball players.

\section{Participants}

Twenty-four participants were selected to take part in this study. Inclusion criteria adopted were as follows: [1] male handball players with at least one year competitive experience, [2] regular participation in training sessions, [3] not smoking, [4] no respiratory and/or cardiovascular diseases, [5] no use of supplements and/or medications that could influence physical performance. Five of the participants withdrew during the experiment because of transfer to other handball teams. Therefore, the final study sample consisted of nineteen participants who completed the study.

The athletes were randomly divided into an experimental group (EG), age: $19 \pm 4$ years, body mass: $79 \pm 13 \mathrm{~kg}$, body height: $1.7 \pm 0.0 \mathrm{~m}$, training experience: $6.8 \pm 3$ years, that maintained their regular sporting activities and carried out IMT with progressive loads $(\mathrm{n}=10)$, and a placebo group (PG), age: $22 \pm 1$ years, body mass: $83 \pm 12$ $\mathrm{kg}$, body height: $1.8 \pm 0.0 \mathrm{~m}$, training experience: $7.5 \pm 3$ years, that also maintained their regular sporting activities, yet additionally carried out IMT with a load considered to be a placebo ( $\mathrm{n}=$ 09).

Participants were first submitted to initial evaluations, with the procedures described in the next section, and reevaluated after 12 weeks of IMT for subsequent analysis and comparison with the initial values. All the players were previously informed about the experimental procedures, and signed a clarified term of consent. The Ethics Committee of the Methodist University of Piracicaba, with the number 62/13, approved the project and it was published in the ClinicalTrials.gov Protocol and Results Registration System: ID (NCT02650882).

\section{Measures}

Respiratory muscle strength and physical performance evaluations were carried out at two occasions: (a) before the start of training; (b) after 12 weeks of IMT. Both measurement sessions were carried out in an air-conditioned room with a temperature between 22 and $24^{\circ} \mathrm{C}$ and relative air humidity of 40 to $60 \%$. The participants were familiarized with the experimental protocol and were instructed not to ingest stimulants (coffee, tea, soft drinks, etc.) or alcoholic beverages before the evaluations, and not to perform any strenuous physical activity in the 24 hours preceding the tests. They were also advised to maintain 
adequate hydration and have a light meal up to 2 hours before carrying out the tests. Prior to the experiment, participants were also interviewed to verify their state of health; they were also verbally questioned by the evaluator about any feelings of discomfort on the day of testing.

Maximal inspiratory pressure (MIP) followed by maximal expiratory pressure (MEP) values were obtained from residual volume and total lung capacity, respectively, with each subject seated in a chair, wearing a nose clip and having a rigid, plastic flanged mouthpiece in place. The participants were connected to a manual shutter apparatus with the maximal pressures measured using a manovacuometer with an aneroid-type gauge $\left( \pm 300 \mathrm{cmH}_{2} \mathrm{O}\right.$; GER-AR, São Paulo, SP, Brazil). A small leak was introduced between the occlusion and the mouth in order to prevent glottic closure, and the subjects also held their cheeks with one hand during testing. Inspiratory or expiratory effort was sustained for at least $1 \mathrm{~s}$. Measurements were taken by a designated technician who always explained and demonstrated the correct performance. Participants completed three to five acceptable and reproducible maximal trials (i.e., differences of $10 \%$ or less between values); the highest value reached was registered for further analysis. An interval of about $1 \mathrm{~min}$ was allowed between efforts. Evaluation methodology and the equations for predicting the normal values of maximum respiratory pressure (MIP and MEP) followed Neder et al. (1999).

MVV tests were carried out with a spirometer (Easy one, ndd Medizintechnik AG, Zurich, Switzerland) according to the American Thoracic Society's (ATS, 2001) guidelines for technique, acceptability, and reproducibility. The device was calibrated before each test according to the manufacturer's instructions. MVV is the largest volume that can be voluntarily breathed into and out of the lungs in a 10- to 15-s interval. In this study, MVV was measured by having the participant breathe deeply (with a volume greater than the tidal volume but lower than the vital capacity) and rapidly for $12 \mathrm{~s}$. The values were recorded in liters per minute by extrapolating the 12 s-accumulated volume to 1 minute. Testing was carried out until 3 acceptable and 2 reproducible curves were obtained without exceeding 8 attempts. During testing, real-time graphics of the curves were provided, indicating whether they met the acceptance criteria proposed by the ATS (2001).

Participants carried out the maximum effort protocol proposed by Lourenço et al. (2011), which consisted of a treadmill incremental maximum oxygen uptake test with gas exchange analysis, used to determine maximal and submaximal variables. Players were familiar with this protocol as it was commonly used in the team physical evaluation during the season. The protocol was carried out on a treadmill with fixed inclination of $1 \%$. The protocol started with a 3 min warm up at $9 \mathrm{~km} / \mathrm{h}$, and the speed was then increased by $0.3 \mathrm{~km} / \mathrm{h}$ every $25 \mathrm{~s}$ until exhaustion. The participants were verbally encouraged to reach the maximum intensity possible for the exercise, and criteria for terminating the test were voluntary exhaustion or reaching the maximum heart rate (considering 220-age). After terminating the test, participants were submitted to a 5 -min recovery protocol, where the speed was reduced every minute to the following values: $60 \%, 55 \%$, $50 \%, 45 \%$ and $40 \%$ of the maximum speed attained. The oxygen uptake $\left(\mathrm{VO}_{2}\right)$, carbon dioxide production $\left(\mathrm{VCO}_{2}\right)$, maximum ventilation (VE) and respiratory exchange rate (RER) were measured breath by breath using the gas analyzer (CPX/D Med Graphics ${ }^{\circledR}$, St. Paul, MN). The heart rate was recorded using a transmission tape and interface (Polar®, Finland). Before each test the gas analyzer was calibrated with a known gas mixture $\left(12 \% \mathrm{O}_{2}+5 \% \mathrm{CO}_{2}\right)$ and the volume sensor with a 3L syringe. The last stage completed by the subjects was considered for the determination of $\mathrm{VO}_{2 \max }$, the maximum speed attained $\left(\mathrm{vVO}_{2 \max }\right)$, the maximum carbon dioxide production $\left(\mathrm{VCO}_{2 \max }\right)$ and maximum ventilation (VE $\mathrm{max}$ ). The V-slope method was used to determine the ventilatory threshold (VT) and the respiratory compensation point (RCP), which allowed for the characterization of the VT and RCP from the loss of linearity of $\mathrm{VCO}_{2} / \mathrm{VO}_{2}$ and $\mathrm{VE} / \mathrm{VCO}_{2}$, respectively.

\section{Design and Procedures}

Experimental procedures were carried out by trained researchers and a qualified team. The evaluations and IMT were conducted after the pre-season, during the competitive season. The training frequency was based on the competition program, which followed the 
traditional annual model.

The inspiratory muscle training program was carried out using an inspiratory muscle exerciser (POWERbreathe - Plus Heavy Resistance Sports model) with the following protocol for the EG: the $1^{\text {st }}$ to the $4^{\text {th }}$ week: intensity of $50 \%$ of the MIP; the $5^{\text {th }}$ to the $8^{\text {th }}$ week: intensity of $60 \%$ of the MIP; the $9^{\text {th }}$ to the $12^{\text {th }}$ week: intensity of $70 \%$ of the MIP. The protocol for the PG was: the $1^{\text {st }}$ to the $12^{\text {th }}$ week: intensity of $15 \%$ of the MEP (HajGhanbari et al., 2013).

Throughout the 12 weeks, two sessions were performed daily, five times a week (preceding specific team training); both groups carried out thirty maximum inspirations, maintaining the diaphragmatic inspiratory muscle pattern (Janssens et al., 2013). In order to guarantee an overload during IMT, the maximum inspiratory pressure values were reevaluated every week using the same protocol described in the evaluation, but the training load was only readjusted for the EG.

All the participants carried out the same volume of specific training for the modality during the 12 weeks of the IMT protocol. Training consisted of five weekly 2-hour sessions, divided into two sessions with emphasis on physical training, and three sessions on handball technical and tactical activities.

\section{Statistical Analysis}

After an analysis of data distribution carried out using the Shapiro-Wilk test, the hypothesis of normality was accepted for all variables. Parametric tests were used to determine the significance of data, by a paired t-test for intragroup comparison, and an unpaired t-test for comparison between the groups. All the results are presented as means plus their respective standard deviations. The level of significance of $5 \%$ was adopted for all analyses and the statistical procedures were carried out using GraphPad InStat software, version 3.05.

With the objective of quantifying the magnitude of the effect of training between the EG and the PG, the effect size was calculated using the Effect Size Generator software, version 2.3 (Swinburne University of Technology, Center for Neuropsychology, Melbourne, Australia), the results being interpreted according to the proposals of Rhea (2004) for trained subjects. The effect size was considered trivial when the result of the calculations was below 0.35 , small when between 0.35 and 0.80 , moderate between 0.80 and 1.50 and large when above 1.5.

\section{Results}

Table 1 presents the results obtained for respiratory muscle strength of each group as well as $p$ value of the comparison. After the experimental protocol, the values for MIP and MEP were significantly larger for the EG. For the EG, the MIP pre $\left(170 \pm 34.3 \mathrm{cmH}_{2} \mathrm{O}\right)$ in comparison with post $\left(262 \pm 33 \mathrm{cmH}_{2} \mathrm{O}\right)$ presented significant increments corresponding to $54 \%$, while for MEP the pre $\left(177 \pm 36 \mathrm{cmH}_{2} \mathrm{O}\right)$ in comparison with post $\left(218 \pm 37 \mathrm{cmH}_{2} \mathrm{O}\right)$ values increased by $23 \%$. For the PG, only MIP showed a significant increase $\left(173 \pm 44\right.$ vs. $\left.213 \pm 21 \mathrm{cmH}_{2} \mathrm{O}\right)$ with an increment of $23 \%$. When the groups were compared post-training, the MIP and MEP values in the EG were significantly higher. In relation to the effect size post training between the EG and the PG, it can be seen that the effect was large for MIP (2.73) and MEP (1.12). As for the maximum voluntary ventilation, there was a significant increase for the EG when comparing pre $(162 \pm 25$ $\mathrm{L})$ and post $(174 \pm 30 \mathrm{~L})$ values, with a small effect size (0.39) between the groups post training.

Table 2 shows the results obtained for by the cardiopulmonary exercise test (CPET). Only for the EG there was a significant increase when comparing pre and post training values for $\mathrm{VO}_{2 \max }$ $(54.0 \pm 8.9 \mathrm{~mL} / \mathrm{kg} / \mathrm{min}$ to $60.0 \pm 7.1 \mathrm{~mL} / \mathrm{kg} / \mathrm{min})$ and oxygen uptake at the RCP $(46.8 \pm 6.7$ $\mathrm{mL} / \mathrm{kg} / \mathrm{min}$ to $50.3 \pm 5.2$ ). In relation to the effect size of training between the EG and the PG post training, the effect was moderate for both $\mathrm{VO}_{2 \max }$ $(0.85)$ and oxygen uptake at the RCP (0.50).

\section{Discussion}

This is the first study to investigate the effect of IMT on handball players. The was to investigate whether 12 weeks of IMT could increase respiratory muscle strength and aerobic performance of handball athletes. The results showed a significant improvement in maximum respiratory pressures, maximum voluntary ventilation, $\mathrm{VO}_{2 \max }$ and oxygen uptake at the $\mathrm{RCP}$ for the EG, while for the PG, only an increase in MIP was observed. 


\section{Table 1}

Comparison of the maximum respiratory pressure values between the PG and EG pre and post IMT. Analysis of the effect of size of the training between the groups

\begin{tabular}{lcccccc}
\hline \multirow{2}{*}{ Variables } & Pre & Post & ES & Pre & Post & ES \\
& & & & & & \\
\hline $\mathrm{MIP}\left(\mathrm{cmH}_{2} \mathrm{O}\right)$ & $173 \pm 44$ & $213 \pm 21^{* \#}$ & 1.14 & $170 \pm 34.3$ & $262 \pm 33^{* *}$ & 2.73 \\
& & & & & & \\
$\mathrm{MEP}\left(\mathrm{cmH}_{2} \mathrm{O}\right)$ & $188 \pm 58$ & $206 \pm 54$ & 0.30 & $177 \pm 36$ & $218 \pm 37^{* *}$ & 1.12 \\
$\mathrm{MVV}(\mathrm{L})$ & $175 \pm 33$ & $181 \pm 25$ & 0.20 & $162 \pm 25$ & $174 \pm 30^{*}$ & 0.39
\end{tabular}

$M I P=$ maximum inspiratory pressure; $M E P=$ maximum expiratory pressure; $M V V=$ maximum voluntary ventilation ${ }^{*} p<0.05$ : comparison between pre and post training; $" p<0.05$ : comparison between the groups post training; $E S=$ Effect of size comparison pre and post training.

\section{Table 2}

Comparison of the variables of the cardiopulmonary test between the PG and EG pre and post IMT. Analysis of the effect size between the groups

\begin{tabular}{|c|c|c|c|c|c|c|}
\hline \multirow[b]{2}{*}{ Variables } & \multicolumn{3}{|c|}{ PG } & \multicolumn{3}{|c|}{ EG } \\
\hline & Pre & Post & ES & Pre & Post & ES \\
\hline $\mathrm{VO}_{2 \max }(\mathrm{mL} / \mathrm{kg} / \mathrm{min})$ & $55.3 \pm 4.5$ & $53.8 \pm 6.1$ & 0.18 & $54.0 \pm 8.9$ & $60.0 \pm 7.1^{* \#}$ & 0.85 \\
\hline $\mathrm{HR}_{\mathrm{VO}} 2 \max (\mathrm{bpm})$ & $191 \pm 7$ & $192 \pm 7$ & 0 & $193 \pm 7$ & $195 \pm 7$ & 0.27 \\
\hline $\mathrm{i} \mathrm{VO}_{2 \max }(\mathrm{km} / \mathrm{h})$ & $14.6 \pm 0.8$ & $14.33 \pm 0.9$ & 0 & $14.9 \pm 1.4$ & $15.6 \pm 0.9{ }^{\sharp}$ & 0 \\
\hline $\mathrm{VT}(\mathrm{mL} / \mathrm{kg} / \mathrm{min})$ & $39.1 \pm 3.2$ & $39.41 \pm 4.1$ & 0 & $39.8 \pm 6.1$ & $40.5 \pm 3.1$ & 0 \\
\hline HR VT (bpm) & $172 \pm 11$ & $169 \pm 10$ & 0.27 & $167 \pm 11$ & $170 \pm 8$ & 0.29 \\
\hline $\mathrm{i}$ VT $(\mathrm{km} / \mathrm{h})$ & $10.9 \pm 0.5$ & $10.8 \pm 0.8$ & 0 & $10.7 \pm 0.5$ & $11.1 \pm 0.5$ & 0 \\
\hline $\mathrm{RCP}(\mathrm{mL} / \mathrm{kg} / \mathrm{min})$ & $45.9 \pm 3.2$ & $46.9 \pm 3.6$ & 0.29 & $46.8 \pm 6.7$ & $50.3 \pm 5.2 *$ & 0.50 \\
\hline HR RCP (bpm) & $180 \pm 8$ & $184 \pm 5$ & 0.55 & $181 \pm 12$ & $186 \pm 9$ & 0.36 \\
\hline i RCP $(\mathrm{km} / \mathrm{h})$ & $12.8 \pm 0.6$ & $12.7 \pm 0.6$ & 0 & $13.0 \pm 1.1$ & $13.5 \pm 0.7^{\#}$ & 0 \\
\hline
\end{tabular}

$V_{2 \max }=$ maximum oxygen uptake; $V T=$ ventilatory threshold; $R C P=$ respiratory compensation point; $H R=$ heart rate; $i=$ intensity. ${ }^{*} p<0.05$ : values between pre and post training; ${ }^{*} p<0.05$ : comparison between the groups in the post training condition. ES = Effect of size comparison pre and post training. 
Regarding the increase in respiratory muscle strength, which is represented with an adaptation to specific inspiratory training, these adaptations would probably contribute to a reduction in inspiratory muscle demands for the same load exercised pre training; furthermore, they may also contribute to the reduction of respiratory effort at the same intensity of exercise. The decrease of the respiratory effort favored the maintenance of athletes' physical performance during activities with greater intensity, once they contributed to the attenuation of the onset of the respiratory fatigue mechanism (Romer et al., 2008) responsible for an increased blood flow to the respiratory muscles and a concomitant decrease in the blood flow to the peripheral musculature, directly influencing physical performance.

Some events are described as precursors of this mechanism that limits physical performance. According to Wells and Norris (2009), respiratory muscle performance is decreased in high-intensity exercises ( $>85 \%$ VO2max) in response to an increment of respiratory work, exercise induced arterial hypoxemia, respiratory muscle fatigue, and dyspnea. These factors occur by increased recruitment of inspiratory accessory muscles during high intensity exercise, with the objective to help the diaphragm, as the efficiency falls due to increased exercise intensity (Polla et al., 2004). The recruitment of accessory muscles continues to reduce the efficiency of respiratory mechanics, resulting in a respiratory asynchrony and causing inefficient ventilation, that will promote the reduction of diaphragm perfusion, while further increasing the need of metabolic requirements and the blood flow to this group of muscles (Mcconnell and Romer, 2004). These associated factors will lead to sensory activation of the central nervous system, thus triggering the activation of the metaboreflex mechanism, which will increase the blood flow to the respiratory muscles and reduce the flow to the limbs in activity that will compromise overall performance. Mcconnel (2009) showed that in studies with different endurance sports such as cycling and rowing, that applied IMT protocols, an improvement in inspiratory muscle strength and physical performance was observed when compared to the placebo group. There is evidence that IMT leads to a reduction in respiratory effort, respiratory work and inspiratory muscle fatigue (Romer, 2002a), factors that will reflect in the improvement of functionality and synchrony of ventilatory mechanics contributing to a decrease in respiratory effort, reduced metabolic and blood flow demand during exercise, especially in high intensity endurance exercises, thus leading to improved performance.

Not only increases in strength, but also increases in respiratory muscles resistance can play an important role in physical performance as improvement of such capacities contributes to better conditioning of the inspiratory musculature, with a consequent decrease in muscular physiological requirements in activities of longer duration, as seen in modalities such as running, rowing, triathlons and cycling (Griffiths and Mcconnell, 2007; Ker and Schultz, 1996; Romer et al., 2002a). The increase in resistance capacity of respiratory muscles, represented by increased MVV, contributes positively to the ability to maintain increased respiratory work at high intensities of the exercise. Sales et al. (2016) showed that higher resistance capacity of inspiratory muscles contributed to the maintenance of blood supply and blood perfusion, which directly influenced the reduction of the metabolic demand of these muscles, leading to better performance and functionality.

Neither of the groups presented any changes in the VT after IMT, possibly due to the fact that adaptations to the VT are determined by the level of effort and fatigue generated by specific physical training (Billat, 2001; Bernardi, 2013). In the assessment carried out prior to IMT, athletes were all in optimum physical condition and both groups were submitted to the same load, intensity and volume of training, thus not generating any adaptive alterations in the VT. On the other hand, specific respiratory training contributed to improved conditioning and respiratory muscle endurance, possibly leading to better ventilatory efficiency at high exercise intensity.

According to Romer et al. (2002b), the ergogenic effects of IMT are related to their direct action on the decrease of inspiratory muscle fatigue, which contributes to a better distribution of blood to the muscles during activity, through the delaye of the metaboreflex mechanism activation, which reduces the respiratory and 
peripheral effort perception in intense exercise. Thus, it seems that better conditioning of respiratory muscles may have positively influenced ventilatory efficiency during specific training of the participants, as well as this combination of training methods may justify the fact that only the EG showed a significant improvement in oxygen uptake in the RCP in comparison to the pre IMT assessment.

Present findings show a significant increase in MIP after IMT for both groups (Table $1)$, although this increase did not induce an improvement in physical performance in the PG. Similar findings were obtained by Hart et al. (2001), who showed an increase in inspiratory muscle strength with no improvement in physical performance, suggesting that increased performance after IMT might be related to greater increments in respiratory muscle strength. An increase in the MIP in the PG can be explained by the fact that training performed even with a $15 \%$ load of the MIP generated increased recruitment of respiratory muscles, thus favoring an improved respiratory pattern, yet did not provide benefits in conditioning of the inspiratory musculature, as observed in the group with incremental loads. This justifies the increase in performance observed only in the EG, that showed an improvement of $54.1 \%$ in MIP, whereas the PG registered an increment of only $23 \%$. Furthermore, previous research has shown that increases in inspiratory muscle conditioning after the inspiratory training period, represented by the rise of MIP, contribute to better ventilatory efficiency and reduction in fatigue of these muscles at high exercise intensities (Mcconnel, 2009). It is important to understand that the greater exposure to global specific exercise is responsible for the improvement in aerobic performance in the EG.

No studies with IMT were found in the literature that included handball players. Considering other intermittent modalities, Nicks et al. (2009) evaluated soccer players and confirmed the ergogenic potential of IMT with regard to inspiratory muscle strength and performance of athletes using a specific intermittent recovery test (the Yo-Yo test). Handball is also an intermittent modality with high cardiac demands (Karcher and Buchheit, 2014). When cardiac demands are at maximum levels, an increase of respiratory work occurs concomitantly. This is an important determinant of vascular conductance to the limbs in activity during exercise (Harms, 1997). At high work levels of respiratory muscles, vasoconstriction of the limbs in activity occurs as previously discussed here, through the activation of the metaboreflex (Hill, 2000). We believe that IMT can be extremely beneficial for these athletes, contributing to improved respiratory muscle conditioning, reduced inspiratory muscle fatigue, and better respiratory mechanical efficiency, as well as decreased respiratory effort during highintensity exercise, especially because of the high intensity of the handball modality.

Moreover, Volianitis et al. (2001) showed beneficial results of IMT (i.e. improved conditioning of inspiratory muscles) in rowing performance. They justified that in rowing there was a high mechanical demand for the upper limbs, related to a doubled overload on the inspiratory muscles, due to the recruitment of these muscles for upper limb movements specific for rowing, and the concomitant recruitment of the same muscle groups to respond to ventilatory requirements at high intensity exercise.

Following the above mentioned research, we believe that in handball, there is a high and double mechanical demand on inspiratory accessory muscles. This overload in throwing athletes has been also described by Hartz et al. (2015), and related to the high recruitment of the trunk and upper limb musculature during the sport-specific movements, high-contact situations and a high number of throws performed during a game (Povoas et al., 2014). Consequently, improvement in inspiratory muscle conditioning may reflect a reduced mechanical demand and fatigue for inspiratory accessory muscles during intensive levels of handball game, which may be another factor associated with enhanced performance.

The improvement in performance of the respiratory muscles could be responsible for the better ventilatory efficiency and greater inspiratory fatigue resistance, contributing to a smaller metabolic demand by this musculature during high intensity exercise. The results achieved following inspiratory muscle training show a potential for preventing or minimizing the activation of the metaboreflex mechanism 
(Oueslatia et al., 2016). We believe that these benefits favored a greater time of exposure to the specific global handball exercise, being the overall exercise responsible for increased aerobic conditioning observed in the athletes.

\section{Conclusion}

The present study showed that 12 weeks of IMT promoted an increment in respiratory muscle strength and resistance as well as improvement in aerobic physical performance in handball athletes. These findings could be applied in sports training as a strategy to minimize the effects of respiratory fatigue in athletes, favoring a greater time in practice and an improvement in overall performance.

\section{References}

American Thoracic Society /European Respiratory Society (ATS/ERS). Statement on Respiratory Muscle Testing. Am J Respir Crit Care Med, 2001; 166: 518-624

Bernardi E. Respiratory Muscle Endurance Training Improves Breathing Pattern in Triathletes. SciMed, 2013; 1(1): 1003

Billat LV. Interval training for performance: a scientific and empirical practice. Special recommendations for middle- and long-distance running. Part II: anaerobic interval training. Sports Med, 2001; 31(2): 75-90

Griffiths LA, Mcconnell AK. The influence of inspiratory and expiratory muscle training upon rowing performance. Eur J Appl Physiol, 2007; 99: 457-66

Harms CA, Wetter TJ, St Croix CM, Pegelow DF, Dempsey JA. Effects of respiratory muscle work on exercise performance. J Appl Physiol, 1997; 89: 131-138

Hart N, Sylvester K, Ward S, Cramer D, Moxham J, Polkey MI. Evaluation of an inspiratory muscle trainer in healthy humans. Respir Med, 2001; 95: 526-53

Hartz CS, Pires PF, Moreno MA, Bigaton DR. EMG frequency spectral characteristics of inspiratory accessory muscles during the shoulder lifting motion in throwing athletes. MTPERehabJournal, 2015; 13: 232

HajGhanbari B, Yamabayashi C, Buna TR, Coelho JD, Freedman KD, Morton TA, Palmer SA, Toy MA, Walsh C, Sheel AW, Reid WD. Effects of Respiratory Muscle Training on Performance in Athletes: A Systematic Review With Meta-Analyses. J Strength Cond Res, 2013; 27(6): 1643-1663

Hill JM. Discharge of group IV phrenic afferent fibers increases during diaphragmatic fatigue. Brain Research, 2000; 856: 240-244

Janssens L, Brumagne S, Mcconnell AK, Raymaekers J, Goossens N, Gayan-Ramirez G, Hemans G, Trooters T. The assessment of inspiratory muscle fatiguein healthy individuals: A systematic review. Respir Med, 2013; 107: 331-46

Karcher C, Buchheit M. On-Court Demands of Elite Handball, with Special Reference to Playing Positions. Sports Med, 2014; 44(6): 797-814

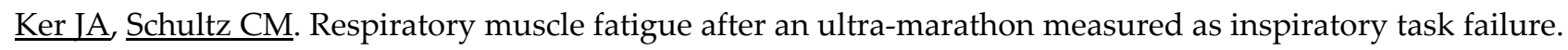
Int J Sports Med_1996; 17(7): 493-96

Lomax M, Tasker L, Bostanci O. An electromyographic evaluation of dual role breathing and upper body muscles in response to front crawl swimming. Scand J Med Sci Sports, 2014; 25(5): 472-8

Lourenço TF, Martins LE, Tessutti LS, Brenzikofer R, Macedo DV. Reproducibility of an incremental treadmill $\mathrm{VO}_{2 \max }$ test with gas exchange analysis for runners. J Strength Cond Res, 2011; 25: 1994-99

Mcconnell AK, Romer LM. Dyspnea in health and obstructive pulmonary disease: the role of respiratory muscle function and training. Sports Med, 2004; 34: 117-132

McConnell AK. Respiratory muscle training as an ergogenic aid. Journal of Exercise Science \& Fitness, 2009; 7(2): 18-27

Neder JA, Andreoni S, Lerario MC, Nery LE. References values for lung function tests. II. Maximal respiratory pressures and voluntary ventilation. Braz J Med Biol Res, 1999; 32(6): 719-27 
Nicks CR, Morgan DW, Fuller DK, Caputo JL. The influence of respiratory muscle training upon intermittent exercise performance. Int J Sports Med, 2009; $30: 16-21$

Oueslatia F, Booneb J, Ahmaidia S. Respiratory muscle endurance, oxygen saturation index in vastuslateralis and performance during heavy exercise. Respiratory Physiology \& Neurobiology, 2016; 227: 41-47

Polla B, D'antona G, Bonttinelli_R, Reggiani_C. Respiratory muscle fibres: specialisation and plasticity. Thorax, 2004; 59(9): $808-17$

Povoas SC, Ascensão AA, Magalhães J, Seabra AF, Krustrup P, Soares JM, Rebelo AN. Physiological demands of elite team handball with special reference to playing position. LStrength Cond Res, 2014; 28(2): 430-42

Rhea MR. Determining the magnitude of treatment effects in strength training research through the use of the effect size. J Strength Cond Res, 2004; 18(4): 918-20

Romer LM, McConnell AK, Jones DA. Effects of inspiratory muscle training on time-trial performance in trained cyclists. J Sports Sci, 2002a; 20: 547-562

Romer LM, McConnell AK, Jones DA. Inspiratory muscle fatigue in trained cyclists: effects of inspiratory muscle training. Medicine and science in sports and exercise, 2002b; 34(5): 785-792

Romer LM, Polkey MI. Exercise induced respiratory muscle fatigue: implications for performance. J Appl Physiol, 2008; 104(3): 879-88

Sales ATN, Fregonezi GAF, Ramsook EH, Guenette JA, Lima INDF, Reid WD. Respiratory muscle endurance after training in athletes and nonathletes: A systematic review and meta-analysis. Physical Therapy in Sport, 2016; 17: 76-86

Stcroix CM, Morgan BJ, Wetter TJ, Dempsey JA. Fatiguing inspiratory muscle work causes reflex sympathetic activation in humans. J Physiol, 2000; 529: 493-504

Volianitis S, McConnell AK, Koutedakis Y, McNaughton L, Backx K, Jones DA. Inspiratory muscle training improves rowing performance. Med Sci Sports Exerc, 2001; 33: 803-809

Wagner H, Finkenzeller T, Würth S, Duvillard SP. Individual and Team Performance in Team-Handball: A Review. J Sports Sci Med, 2014; 13: 808-816

Wells GD, Norris SR. Assessment of physiological capacities of elite athletes and respiratory limitations exercise performance. Pediatric Respiratory Reviews, 2009; 10: 91-98

\section{Corresponding author:}

\section{Marlene Aparecida Moreno}

Universidade Metodista de Piracicaba, Rodovia do Açúcar, km 156 (SP-308)

13.423-170- Piracicaba, SP, Brazil.

Phone: (+55)(19) 31241558.

E-mail: ma.moreno@terra.com.br 